\title{
Retrospective Evaluation of Different Shade Selection Methods in the Context of the Vital Bleaching Technique
}

\author{
Ezgi Tuter Bayraktar ${ }^{1}\left(\mathbb{D}\right.$, Zuhre Hale Cimilli ${ }^{2}\left(\mathbb{C}\right.$, Nevin Kartal ${ }^{2}\left(\mathbb{D}\right.$, Cafer Turkmen ${ }^{2}$ \\ ${ }^{1}$ Private Practice, Istanbul, Turkey. \\ ${ }^{2}$ Department of Restorative Dentistry, Faculty of Dentistry, Marmara University, Istanbul, Turkey.
}

Correspondence Author: Ezgi Tuter Bayraktar

E-mail: tuterezgi@gmail.com

Received: 18.01.2021 Accepted: 14.04 .2021

\begin{abstract}
Objective: This retrospective study was performed to evaluate the correspondence between digital image analysis and spectrophotometry tooth shade results.

Methods: The study population consisted of 10 patients. Office bleaching was performed using an in-office whitening. Tooth shade was analysed by spectrophotometry and image analysis of digital photographs at baseline, and at 1-week and 3-month follow-up visits. The correspondence between the two methods was evaluated based on the total color change $(\triangle \mathrm{E})$, calculated using lightness, chroma, and hue (LCH) values for spectrophotometry and lightness, red-green, blue-yellow (LAB) values for digital image analysis. The Mann-Whitney U, Wilcoxon signed rank and Kruskal-Wallis tests were used for the statistical analyses $(p<0.05)$. The correspondence between digital image analysis and spectrophotometry results was determined using the intraclass correlation coefficient (ICC).

Results: At the 3-month follow-up, there were no significant differences in tooth shade results between the spectrophotometry and digital image analysis methods ( $p=0.855$ ), which showed strong agreement (ICC $=0.983$ ).

Conclusion: Digital image analysis may be a useful alternative to spectrophotometry for tooth shade selection.

Keywords: Bleaching, Digital image analysis, Shade Selection, Spectrophotometer
\end{abstract}

\section{INTRODUCTION}

Dental esthetics is an important issue for all dental practices. Well-aligned white teeth are desired by the majority of both women and men. People desire the "perfect smile", and dental bleaching represent the initial step towards this goal.

Vital bleaching techniques have proven efficacy, either in the office or at home under professional supervision. Home and in-office bleaching techniques utilize peroxide at different concentrations (1). In-office whitening agents use a higher concentration of peroxide and provide more rapid results. Therefore, patients frequently request in-office bleaching. Clinical application often involves a combination of two bleaching techniques for rapid whitening with less dental sensitivity (2).

High concentrations (25-40\%) of hydrogen peroxide $\left(\mathrm{H}_{2} \mathrm{O}_{2}\right)$ enhance tooth colour (3). In-office whitening performance is related to the ability of $\mathrm{H}_{2} \mathrm{O}_{2}$ to diffuse into the hard tissues of the teeth, although deeper penetration is associated with a higher risk of pulp damage. Improvements in whitening techniques could decrease the detrimental effects on pulp tissue with no decrease in efficacy (4).

Shade selection is done using visual or instrumental techniques. The visual technique is the most commonly used for shade selection by dentists but is subjective and depends on numerous environmental factors and the vision of the clinician (5). Instrumental techniques using colorimeters, spectroradiometers, spectrophotometers and digital cameras are objective and convenient for shade selection in clinical practice (6).

Spectrophotometry, which measures the transmittance curve or spectral reflectance of a specimen, quantifies the amount of light reflected from the surface in the visible spectrum at each wavelength and is the most appropriate method for shade selection. Spectrophotometers are unaffected by metamerism and have a longer working life than colorimeters (7). 
Digital image analysis is a shade selection method based on digital photography. Shade selection using this method can simplify communication between the technician and the dentist. Digital image analysis requires an appropriate software program to analyse colour values in an appropriate format. Digital image analysis is a cost - and time-effective method compared to spectrophotometry and colorimetry, although its validity has not yet been proven (8).

In the present study, consistency between spectrophotometric and digital image analysis tooth shade selections, at 1 week and 3 months after in-office bleaching, was evaluated. The null $\left(h_{0}\right)$ hypothesis was that the digital image analysis and spectrophotometric measurements would not be consistent.

\section{METHODS}

This retrospective clinical study was carried with approval from the Marmara University Faculty of Dentistry Ethics Committee (Istanbul, Turkey; registry no. 2020-383).

The data of patients treated with in-office bleaching procedures at Marmara University, Faculty of Dentistry, Restorative Department, between January 1, 2019, and January 1, 2020 were analysed. The study population in this retrospective analysis consisted of 10 patients aged between 20 and 35 years. Participants were examined in the context of the exclusion/inclusion criteria.

The inclusion criteria were regular attendance of follow-up appointments, caries-free anterior teeth, no restorations on the labial surface, and good general and oral health. The exclusion criteria were pregnant or lactating women, active carious lesions, dentine exposure, endodontic treatment of anterior teeth, internal tooth discolouration, and current smoking.

The bleaching procedure was performed using Opalescence Xtra Boost (Ultradent Products, South Jordan, UT, USA) in two 20-minute sessions, conducted in accordance with the manufacturer's instructions.

Tooth shade was assessed based on follow-up appointment data. Total colour change $(\Delta \mathrm{E})$ was denoted by $\Delta \mathrm{E}_{1}$ (baseline and 1 week) and $\Delta E_{2}$ (baseline and 3 months) and assessed using both spectrophotometry and digital image analysis. Measurements were performed for teeth numbers 11 and 13 , from the middle third of the labial surface.

\section{1. $\Delta E$ Measurements Obtained with Spectrophotometry}

Shade selection was performed by one clinician using a Vita Easyshade V spectrophotometer (Vita Zahnfabrik, Bad Säckingen, Germany). The device was calibrated for each measurement according to the manufacturer's instructions (Figure 1). Lightness, chroma and hue (LCH) values from $\mathrm{CIELAB}$ colour space were determined and $\triangle \mathrm{E}$ was calculated (9).

\section{2. $\Delta E$ Measurements Obtained with Digital Image Analysis}

A Canon EOS 700D digital single lens reflex (DSLR) digital camera (Canon, Melville, NY, USA), macro lens (100 mm; Canon), and macro twin flash (Yongnuo YN-24EX/TTL; Shenzhen Yong Nuo Photographic Equipment, Shenzhen, China) were used for the study. Lightness, red-green, blueyellow ( $L A B$ ) values were determined with Adobe Photoshop CC software (Adobe Systems Inc., San Jose, CA, USA; Figure 2) and $\Delta E$ values were then calculated using a previously described formula (9).

$\Delta \mathrm{E}=\mathrm{v}\left[\left(L_{1}^{*}-\mathrm{L}_{0}^{*}\right)^{2}+\left(\mathrm{a}_{1}^{*}-\mathrm{a}_{0}^{*}\right)^{2}+\left(\mathrm{b}_{1}^{*}-\mathrm{b}_{0}^{*}\right)^{2}\right]$

The normality of the data was analysed using the Kolmogorov-Smirnov test. The two methods were compared with the Mann-Whitney $U$ test. Measures were compared among three appointments using the Wilcoxon signed rank test. The intraclass correlation coefficient (ICC) was used to evaluate the agreement between spectrophotometry and digital image analysis tooth shade results $(p<0.05)$.

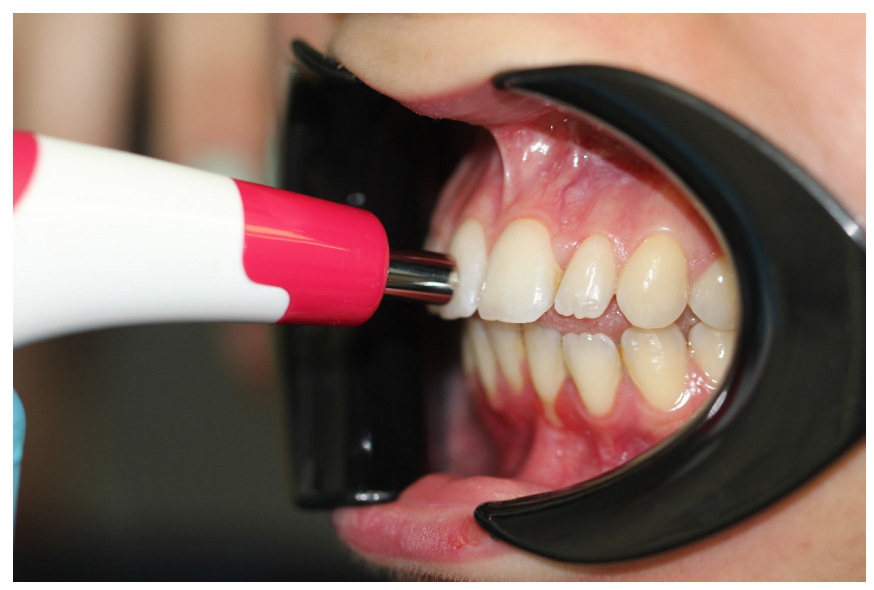

Figure 1. Determination of $\mathrm{LCH}$ values with spectrophotometer

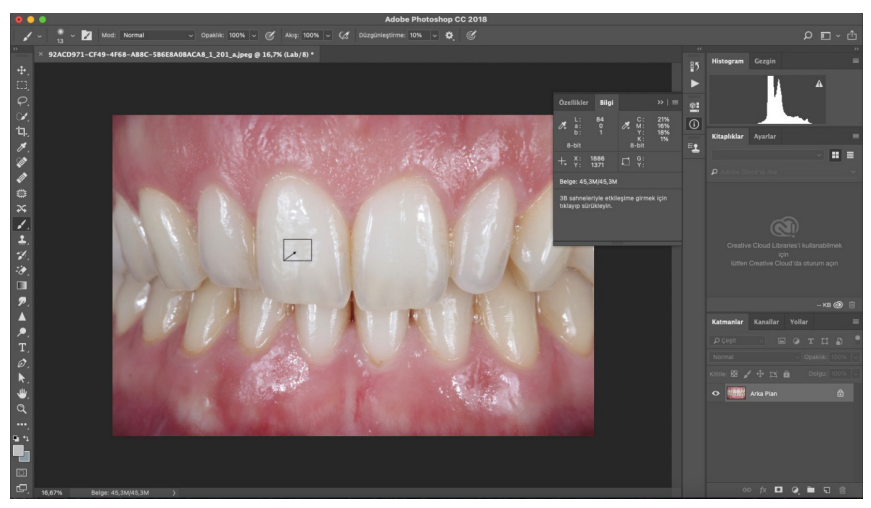

Figure 2. Determination of $L A B$ values with digital image analysis

\section{RESULTS}

The shade selection accuracy was compared between the digital image analysis method and spectrophotometry. 
The difference in $\Delta \mathrm{E}$ values between spectrophotometry and digital image analysis was not statistically significant $(p=0.855)$ (Table 1). The two methods showed a high degree of agreement (ICC=0.983).

Table 1. $\Delta E$ Values of two different methods for all measurements

\begin{tabular}{lllll}
\hline & Mean \pm SD & Median & Q1 - Q3 & $p$ \\
\hline Digital Image Analysis & $4.00 \pm 2.01$ & 3.43 & $2.53-4.84$ & \\
& & & & 0.855 \\
Spectrophotometer & $4.08 \pm 1.99$ & 3.66 & $2.54-5.07$ & \\
\hline
\end{tabular}

There were no statistically significant differences between the median spectrophotometric and digital image analysis $\Delta \mathrm{E}_{1}$ values $(p=0.808)$ or $\Delta \mathrm{E}_{2}$ values $(p=0.957)$ (Tables 2 and 3$)$.

The 1-week and 3-month follow-up data were analysed separately. The mean $\Delta \mathrm{E}$ value obtained using spectrophotometry at the 3-month follow-up was 4.58 . Values equal to or above the $\Delta \mathrm{E}$ threshold of 3.3 are considered noticeable by clinicians (10), so the colour change was clinically detectable in this study.

Table 2. $\triangle E 1$ values of two different method

\begin{tabular}{lllll}
\hline & Mean \pm SD & Median & Q1-Q3 & $p$ \\
\hline Digital Image Analysis & $3.13 \pm 1.04$ & 3.06 & $2.4-3.4$ & \\
& & & & 0.808 \\
Spectrophotometer & $3.30 \pm 1.26$ & 2.88 & $2.4-3.7$ & \\
\hline
\end{tabular}

Table 3. $\triangle E 2$ Values of two different method

\begin{tabular}{lllll} 
& Mean \pm SD & Median & Q1-Q3 & $p$ \\
\hline Digital Image Analysis & $4.88 \pm 2.36$ & 4.53 & $3.28-6.84$ & \\
& & & & 0.957 \\
Spectrophotometer & $4.88 \pm 2.30$ & 4.58 & $3.27-6.6$ & \\
\hline
\end{tabular}

\section{DISCUSSION}

The present study was performed to determine whether there is a linear relationship between spectrophotometry and digital analysis tooth shade results. The main objective was to investigate the practical utility of digital colour analysis systems in dentistry. Both methods have their own algorithm for colour analysis, and separate $\Delta E$ values were obtained for each method. The $\Delta \mathrm{E}$ values were subjected to correlation analysis, and the $h_{0}$ hypothesis that the digital image analysis and spectrophotometric measurements would not be consistent was rejected (i.e. high correspondence was found between the two methods).

Patients' expectations regarding esthetic outcomes are increasing, and esthetic treatments are being performed more widely, including bleaching (11). Tooth colour was not measured immediately after the bleaching session in this study, because dehydration of the tooth can affect the measurements. Thus, shade analysis was performed 1 week and 3 months after the bleaching treatment. The effectiveness of bleaching increases with the application of highly concentrated $\mathrm{H}_{2} \mathrm{O}_{2}$ products over two sessions (12). Clinically significant colour improvement is also observed. Visual shade selection is the most commonly used method by clinicians. However, this technique is affected by a number of variables and is subjective (13). Therefore, an instrumental alternative method was developed in the late 1990 s. Shade detection instruments, such as colorimeters and spectrophotometers, have subsequently been introduced into dentistry. Researchers have reported equivocal outcomes when using colour-measuring devices, with some authors suggesting that these devices do not provide reliable results (14), and others reporting good accuracy (15).

A number of dental photography and software systems have been developed, and digital photography has started to be used for shade selection (16). Digital images can be analysed using convenient software programs that allow all pertinent details to be recorded. This represents a major advantage over contact-type instruments. However, the factors affecting the results obtained with this method, such as the digital camera parameters, reproducibility of digital images, and lighting conditions, should be investigated. More research is also required for standardization of the measurements. The CIELAB colour space is three-dimensional, with L*A*B* axes. A colour stimulus is indicated by chromatic $A^{*}$ (red/ green), $B^{*}$ (yellow/blue) and $L^{*}$ (achromatic) values. Shade differences can be quantified using the CIELAB formula based on Euclidean intervals, which has been used widely to determine colour differences in both dentistry and the industrial sector. CIE created a new colour formula, known as CIEDE2000, which is more complex than the CIELAB colour space. However, both of these formulae are convenient for use in clinical practice (17).

Browning et al. reported a Yonghua significant correlation between CIELAB and CIEDE2000 $\triangle E$ values (18). On the other hand, while Oliveria et al. reported that CIELAB and CIEDE2000 $\triangle E$ values were similar, they nevertheless showed some differences, attributed to the use of two different formulae (19). The CIELAB formula was used in the present study.

There have been few reports comparing digital image analysis and spectrophotometry tooth shade results. Further research is therefore needed.

\section{CONCLUSION}

Within the limitations of this retrospective clinical study, we showed that spectrophotometry and digital image analysis yielded similar tooth shade results. Thus, digital cameras can be used for shade analysis and selection and can also facilitate communication between dentists and technicians. 


\section{REFERENCES}

[1] Machado LS, Anchieta RB, dos Santos PH, Briso ALF, Tovar N, Janal MN, Coelho PG, Sundfeld RH. Clinical comparison of athome and in-office dental bleaching procedures: a randomized trial of a split-mouth design. Int J Periodont Restor Dent 2016; 36:251-260.

[2] Dawson PFL, Sharif MO, Smith AB, Brunton PA. A clinical study comparing the efficacy and sensitivity of home vs combined whitening. Oper Dent 2011; 36:460-466.

[3] Cintra LTA, Benetti F, Ferreira LL, Gomes-Filho JE, Ervolino E, Gallinari MO, Rahal V, Briso ALF. Penetration capacity, color alteration and biological response of two in-office bleaching protocols. Braz Dent J 2016; 27:169-175.

[4] Soares DG, Basso FG, Pontes ECV, Garcia LFR, Hebling J, Costa CAS. Effective tooth-bleaching protocols capable of reducing $\mathrm{H}_{2} \mathrm{O}_{2}$ diffusion through enamel and dentine. J Dent 2014;42:351-358.

[5] Ragain JC. A review of color science in dentistry: shade matching in the contemporary dental practice. J Dent Oral Disord Ther 2016; 4:1-5.

[6] Joiner A. Tooth colour: a review of the literature. J Dent 2004; 32:3-12.

[7] Kalantari MH, Seyed AG, Mohaghegh M. Evaluation of accuracy of shade selection using two spectrophotometer systems: vita easyshade and degudent shadepilot. Eur J Dent 2017; 11:196200.

[8] Bengel WM. Digital photography and the assessment of therapeutic results after bleaching procedures. J Esthet Restor Dent 2003;15:21-32.

[9] Vichi A, Louca C, Corciolani G, Ferrari M. Color related to ceramic and zirconia restorations: a review. Dent Mater 2011; 27:97-108.

[10] Francis G, Acharya SR, Sandya K. To determine tooth discolouration after treatment with various endodontic materials using spectrophotometric analysis-an in-vitro study. J Clin Diagn Res 2019; 13:7-12.

[11] Ozdogan A, Duymus ZY, Ozbayram O, Bilgic R. Effect of different bleaching agents on the surface roughness and color stability of feldspathic porcelain. Braz Dent Sci 2019; 22:213-219.

[12] Martins IEB, Onofre S, Franco N, Martins LM, Montenegro A, Arana-Gordillo LA, Reis A, Loguercio AD, Silva LM. Effectiveness of in-office hydrogen peroxide with two different protocols: a two-center randomized clinical trial. Oper Dent 2018; 43:353361.

[13] Jain A, Jain S, Verma N, Parmar D, Parmar S, Surjani P. Assessment and comparison of reliability of conventional visual shade and spectrophotometer shade matching systems in fixed prosthetic rehabilitations. J Res Adv Dent 2019; 9:240245.

[14] Yap AU. Color attributes and accuracy of vita-based manufacturers' shade guides. Oper Dent 1998; 23:266-271.

[15] Ballard E, Metz MJ, Harris BT, Metz CJ, Chou JC, Morton BDS, Lin WS. Satisfaction of dental students, faculty, and patients with tooth shade-matching using a spectrophotometer.J Dent Educ 2017; 81:545-553.

[16] Miyajiwala JS, Kheur MG, Patankar AH, Lakha TA. Comparison of photographic and conventional methods for tooth shade selection: a clinical evaluation. J Indian Prosthodont Soc 2017; 17:273-281.

[17] Westland, S. Review of the cie system of colorimetry and its use in dentistry.J Esthet Res Dent 2003; 15:5-12.

[18] Browning WD, Contreras-Bulnes R, Brackett MG, Brackett WW. Color differences: polymerized composite and corresponding vitapan classical shade tab. J Dent 2009; 37:34-39.

[19] De Oliveira DCRS, Ayres APA, Rocha MG, Giannini M, Rontani RMP, Ferracane JL, Sinhoretti MAC. Effect of different in vitro aging methods on color stability of a dental resin-based composite using cielab and ciede 2000 color-difference formulas. J Esthet Res Dent 2015; 27:322-330. 